\title{
The implications of behavioural economics for pricing in a world of offer optimisation
}

\author{
Octavian Oancea ${ }^{1}$
}

Received: 14 November 2020 / Accepted: 21 January 2021 / Published online: 22 June 2021

(c) The Author(s), under exclusive licence to Springer Nature Limited 2021

\begin{abstract}
The impact of the COVID-19 on the industry has highlighted once again the need to create new ways of addressing consumers that would be less reliant on historic data, and more adjusted to recent observations of consumer behaviour, real time experiments and competitive context. This article addresses the implications of consumer behavioural economics in the design of offers and the way airlines will have to rethink the way they price in a New Distribution Capability (NDC) world, as defined by IATA. In a pandemic impacted era, this investment will show its fruits not only after, but also during the recovery phase, where travel behaviour is exceptionally different, capacity fluctuates and the competitive landscape is equally difficult to predict. We will discuss what challenges a pricing department is facing in this new world of offers, as well as how to overcome them in order to provide relevant and personalised offers to each consumer and price them according to the value that these offers bring. In the COVID-19 context as well as post-pandemic, airlines will need to not only respond quicker with capacity adjustments, but also to better understand human behaviour relative to a purchase decision and address each consumer with an optimised offer in real-time, as they request it. Some of the questions for which new artificial intelligence experimentation techniques and behavioural economics models will require significant development are: What drives a person to choose or not to choose a certain offer? How many choices should they see? What trip purpose segment do they belong to? What is their willingness to pay? What attributes of an offer are people considering and what value do they associate to these offers?
\end{abstract}

Keywords Pricing $\cdot$ Behavioural economics $\cdot$ Revenue management $\cdot$ Innovation $\cdot$ Offer optimisation

\section{Introduction}

The severe COVID-19 impact on the aviation industry is due not only to its sudden appearance and its magnitude, but primarily due to its length. Over the years, airlines have perfected models aiming to adjust/protect their demand forecasting patterns used in revenue optimization in case of a special event, but under the assumption that such an event would occur rarely and will be short in duration. It is one more argument for transforming the way airlines build their products and engage with consumers. Being agile enough to act upon recent trends and to optimize and generate realtime offers with direct control of the distribution is far more relevant in today's context than relying on solid historical

Octavian Oancea

omifam@yahoo.com

1 Geneva, Switzerland data to first forecast demand on a fixed set of previously distributed prices, as it was traditionally designed.

As airlines are gradually transforming themselves into retailers for travel related products and services, the methods used to understand customer preferences and respond to their needs with appropriate offers in real time will substantially influence which market players will survive in the years to come, in a highly competitive industry, where profit margins remain significantly low and cashflow has been dramatically hurt by the pandemic. The IATA New Distribution Capability (NDC) initiative is designed with the goal of enabling airlines to regain control of their revenues by facilitating a new standard of distributing 'rich content', including personalised offers, to all distribution channels, including indirect ones, such as travel agents (Westermann 2013). In this new world, the way an airline will construct and display its offers and the way it can understand and address each consumer behaviour will be of utmost importance over the traditional way of simply optimising the ticket prices as it is done today, 
given an input limited to demand forecast, pre-set prices and capacity data.

In the new world of airline retailing, revenue management needs to address the joint pricing and assortment optimisation problem. Regulatory implications of segmented and continuous pricing will also have to be addressed, as with IATA's NDC, different customers, either as segments or as individuals, could receive different prices for the same product, and those prices could be determined dynamically on a continuous spectrum by the airline without requiring to pre-publish a fixed grid of limited fare levels and rules.

Ancillary revenues should not be managed in isolation of ticket revenues. Simulated results in Passenger Origin-Destination Simulator (PODS) tests have shown that in some cases, bundling a flight with valued ancillary services can increase total net revenues rather than selling the ancillary as optional add-on, and that there is a first mover advantage for the airlines that implement dynamic offer engines ahead of their competitors. It was also shown that price segmentation between passenger types, such as business vs. leisure, can increase revenues (Wang 2020).

\section{Context affects choice}

Traditionally, airlines used to optimise their revenues based on demand forecasts, pricing data, time left and seats left, without necessarily directly observing the competition position as an input to their optimiser. In more recent years, the revenue systems were enriched and competitive context was used not only to set prices, but also to steer availability.

Many advanced airline price optimisation models that use competitive information as an input assume that consumers are perfectly rational given a choice set and would therefore appropriately consider travel options based on price and quality of itinerary. In a pandemic context, the violation of this rationality assumption is further magnified by the various travel policies and restrictions, the capacity adjustments airlines respond with and the human reactions to recent news. Many aspects of behavioural economics are traditionally overlooked and optimisation is executed in a deterministic approach rather than by balancing revenue maximisation with the exploration of human behaviours in the various contexts in which choices are made. In order to be perfectly rational in the new world of offers, where price and quality of itinerary are no longer the only dimensions, people would have to have complete information, would have to be driven by cognition rather than emotion in their decision making and would have to have a very high computational ability to weigh all the elements of the offers they receive. They would also have to show consistency with regards to the axioms of choice such as Completeness-for all $X$ and $Y$, we have $X \geq Y$, or $X \leq Y$ or indifference, Continuity-where there exists some probability such that a gamble between the best and the worst outcome is as attractive as receiving a sure intermediate outcome and Transitivity-if $X \geq Y, Y \geq Z$, then $X \geq Z$. For example, when looking for a travel option from an origin to a destination, a perfectly rational consumer would have to be able to observe and fully understand all available options, including ancillary services and bundles, and be able to rank all of them appropriately based on preference. However, people are far from being econs, defined as unboundedly rational, and are also driven by emotions, previous experiences or social biases (Welch 2019). The compromise effect is noticed in many choice behaviours, as options tend to get chosen more often when they are the compromise option in a choice set.

Over the years, airlines have invested a lot in creating loyalty programs to entice customers to return to the same airline, or to spend more in order to reach a certain tier status. Maintaining such status is also the source of mileage runs, when consumers either upgrade or fly more than originally desired simply to obtain or maintain a certain tier. The endowment effect states that people will more likely pay to preserve things they already own over paying for items that are available but that they do not yet own (Weaver and Frederick 2012). This can also mean that maintaining a certain loyalty tier membership could appear as more valuable to a consumer than a more affordable option they might observe at the time of choosing a travel alternative. Numerous experiments conducted by Weaver and Frederick (2012) showed that manipulations that reduce the gap between valuations and reference prices reduce or eliminate the endowment effect. These results suggest that the endowment effect is often best construed as an aversion to bad deals rather than an aversion to losing possessions.

\section{The new world of offers}

As airlines gather more and more data from their customers, they are able to better understand purchase behaviours and travel patterns and thereby define offers based on refined customer trip segmentation models. It is also easier to enrich their loyalty programs with third party services and offers as well as to create personalised offers based on historical and present interactions between consumers and the airline. However, a sophisticated trip segmentation model, coupled with a dynamic offer generation model that would decide in real time from a vast range of ancillaries which ones to offer a-la-carte and which ones to bundle, including third party options, can generate very complex choices (Wang 2020).

The behaviours people express during/post a pandemic, as well as when dealing with complex choices in fierce competition environments, highlights the limits of specialised and mathematically based solutions that have reigned for so 
long in the revenue management space. With more customer touchpoints than in the past, airlines can become more and more informed of customer preferences and decisions, and can compare what has happened to what was supposed to happen based on the forecasting models used.

Whether sometimes less aware or in other times very well expressed, people use mental accounting techniques and are dynamically creating budgets to judge their expenses. In order to incorporate behavioural economics into their offers, airlines and travel companies could also consider the basis of categorising budgets. For instance, sources of income play a major role in the ways money is consumed. Income from salaries is viewed and spent differently than gift money, and in a pandemic context that thinking is again significantly transformed. Therefore, offering private travel promotions to a company that just issued bonuses to its employees may prove to be much more efficient than regular sales strategies. In addition, budgets are set as a function of spending category. For example, some consumers have a mental account budget for each of the travel components such as their flight ticket, hotel, duty-free, tours, meals, to name a few. A travel company could therefore try to tailor offers to suit such budgets and address more of these categories either directly or by partnering with other service providers. Trying to extract as much as possible from only one budget, such as the flight ticket, is limiting and is not in the long term interest of a travel company aiming to maximise future net profit value. The passage of time is also a big influencer, as some budgets are time-bounded-weekly, monthly or annually. As an illustration, an airline might observe that many customers are not willing to spend $\$ 1500$ on a Business class ticket however they will purchase an Economy ticket and much later on are willing to buy additional services such as upgrades which eventually equal the same product and amount as that which they originally declined. Therefore, a "No" to an ancillary while booking the ticket might translate into a "Yes" later in the pre-travel cycle. Increasing customer touchpoints and breaking expenses into individual transactions has also proven to increase customer engagement (Nammir et al. 2012).

Therefore, a successful new world of offers means an environment in which models automatically define and adjust appropriate dynamic bundling vs. a la carte options, including dynamic prices, in order to generate incremental revenue, based on either identified person or customer segment. This environment considers the request, the inventory left of seats and services at the time of the request, the competition landscape, the time left until departure, the forecasted demand to come and estimated willingness to pay. This means taking into consideration human behaviour particularly in terms of how many additional items should be displayed and how to define the best value proposition of each bundle in a way that can be easily consumed and evaluated by people, while experimenting with the way offers are presented to the consumer (Fig. 1).

For each request, the offer engine determines whether it is optimal to display certain ancillaries à-la-carte or bundle them, based on three elements-its internal data (such as historical observations, costs, inventory position), the offer request details (such as the date of travel, the number of passengers and other trip related information, loyalty member number, if applicable, in the context of personalised offers),

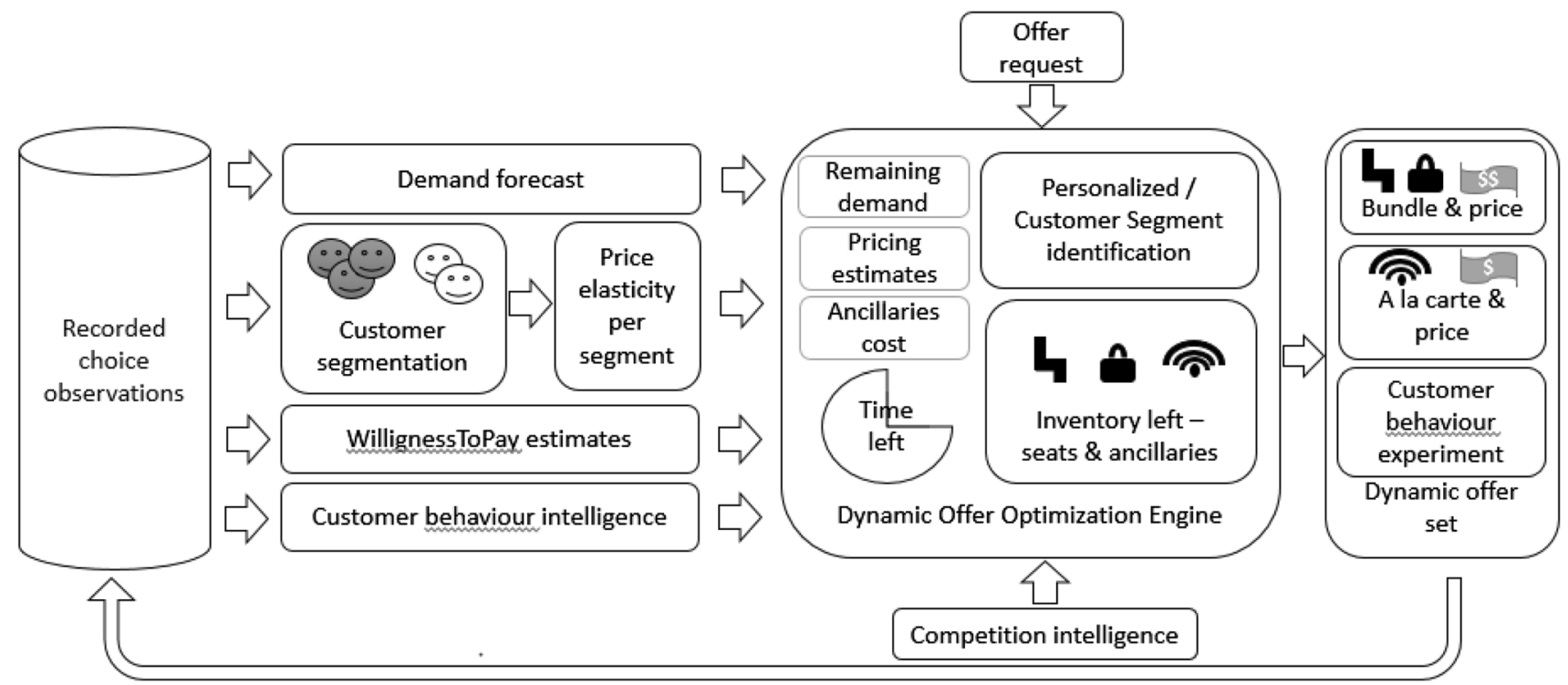

Recorded response to the offer

Fig. 1 Dynamic offer optimisation engine 
and competition information. Although for one request the response could be one bundle, or one standard seat with a few à-la-carte ancillaries, it is more likely to see airlines continuing to give a combination of both-a few bundles to choose from which reflect the various fare brands of the airline, as well as a few ancillaries as optional services. The engine also prices the offers dynamically. The recorded answers to the offers sent are stored and used to further refine the customer trip segmentation models, demand forecasting models, willingness to pay estimates, as well as to prepare the next set of behavioural experiments. In a pandemic context, this could mean appropriately bundling and pricing attributes that have higher utility for consumers, such as flexibility and refundability, priority boarding, extra space, neighbour free seat and wellness support-PCR tests.

Currently, airlines have more flexibility to carry out machine learning experiments with ancillary pricing as they are mostly sold through direct distribution channels. More time is needed to see the same flexibility in the industry with bundles or with ticket pricing. A significant change over the current way of setting fixed prices would be enabling continuous pricing in all distribution channels, limited today by the number of reservation booking designators and published in batches through organisations such as the Airline Tariff Publishing Company (ATPCO) that further distribute the pricing data to Global Distribution Systems.

Should the consumer choose to identify themselves upon the offer request, this would allow for personalised dynamic offers where travel history and stored preferences are used to respond uniquely to the request originator. The trip segmentation models remain useful even in the context of personalised offers. Dependent upon the travel request, the same identified customer can be mapped to a more leisure or more business segment, and therefore observe a trip-tailored offer being displayed, enriched also by the fact that customer preferences are known. When the consumer is unknown, the request is mapped to a customer segment based on the available travel request characteristics-such as advance purchase, length of stay and number of travelers. It is essential for the airline to estimate as accurately as possible the price elasticity of each customer segment that it is trying to address in order to improve its revenues. This means lowering the price only when sufficient additional volume can be generated from that customer segment, or by charging the amount that consumers in that segment are ready to pay for the flight ticket as well as the ancillary services offered. The trip segmentation models require to be updated based on recent trends, as travel purpose patterns change significantly during/post a pandemic, economic downturn/ recovery or political events. For instance, COVID-19 time has seen mostly one way traffic, and VFR-visiting friends and relatives-oriented purposes, with much less business, roundtrip traffic or group travel, as people increasingly worked remotely, met virtually and with most public events being cancelled or deferred.

Bockelie and Belobaba (2017) defined two types of customers based on the ancillary choice model: Simultaneousthose that select the ancillaries at the time of purchasing the itinerary, using an overall willingness to pay for the trip, and Sequential - those that first choose an itinerary and later on evaluate ancillaries. As long as ancillary revenue management remains outside of the optimisation that calculates how many seats should be protected and at which fare level, this can be a significant weakness. In some scenarios, it could even negatively impact the total revenues, if the revenue management system is not adapted to simultaneous passengers ( $\mathrm{Lu}$ 2019). The overall goal of an airline revenue management has to be to maximize its total revenues, by providing to customers the most revenue enhancing assortments and associated prices.

\section{Nudging the consumer: choosing " $B$ " rather than " $A$ "?}

There are few widely recognised commercial strategies used in influencing choice behaviour: restriction on choices (such a product unavailability), discounts and promotions, advertising, and one of the most powerful, nudging-deciding on a choice architecture that will make "B" more appealing than "A". Nudges are not mandates. For example, highlighting the option of a travel bundle with a generously discounted baggage allowance to attract attention and increase likelihood of getting chosen, counts as a nudge. Imposing a fee or a surcharge does not.

One effective technique of nudging is exploiting the sunk cost effect. People are driven to consume what they have prepaid for (Thaler and Sunstein 2009). Imagine someone prepaid for a travel voucher that expires soon. Even if bad weather is forecasted or other such inconveniences, the voucher holder will still be inclined to use it-to get their money's worth. Therefore, when a travel company wishes for people to consume its products or services it could be enough to simply highlight the cost they have already incurred. A sunk cost can be seen as an "investment" for future consumption. During a pandemic impacting dramatically airlines' cashflows, such as the COVID-19, many airlines have created way of incentivizing customers to pre-purchase memberships/vouchers useful for discounting future travels. Thaler (2019) describes two types of consumer utility: acquisition utility - the net amount of well-being a person obtains after subtracting the opportunity cost of a good, and the transaction utility - the difference between the price actually paid and the price one expected to pay-the reference price. Transaction utility can therefore indicate a fair price, a bargain, or an overpriced service. Both utilities are widely spread in sales 
tactics, as some use "suggested retail price" to manipulate the reference price. According to Thaler (2019), people, are time inconsistent and not fully rational by nature. They change between mental accounts of what is preferred now or later on the basis of new information, novel situations, and changes in income. A pandemic context is also magnifying this effect, with sudden travel restrictions/bans being announced or lifted, influencing travel decisions.

There are also ways to weaken the sunk cost effect: by decoupling - physical formats of the transaction (e.g., tickets/vouchers) make it difficult to identify the cost of each consumption opportunity; by depreciation - as time passes the pain of the past payment reduces; and by the inability to meter accurately through currency-when presented with an unfamiliar currency, the difficulty of accurately metering the cost increases. People may also treat foreign currency as "free money", while with local currency people are less likely to spend as much. For example, flying back from a foreign country, travelers look for last minute ways to spend all foreign currency they still hold such as purchases in duty-free shops or on board the aircraft.

As highlighted by Rodway et al. (2012) and as observed by the author of this article through a survey method conducted with over 300 respondents, techniques such as asymmetric dominance/decoy are proven to be very effective in nudging customers towards a certain option in a choice set. Additionally, adding a third choice to a set of two options can sometimes change the way consumers evaluate the first two choices. In the survey conducted, respondents chose from displayed sets of two or three bundles. The results showed that the second bundle is selected more often when a third option is displayed, even when the third option is only there as a decoy-designed to be slightly less appealing than the second bundle (Fig. 2). At equal prices, the preference for option "A" drops from 41 to $26 \%$ and "B"'s preference increases from 59 to $70 \%$, when option "C" is introduced (Scenarios 1 and 2). In Scenario 3, as "B" is now priced lower, "A"s preference drops from 41 to $29 \%$, and falls further to $21 \%$ when the third option is added. Similarly, when "B" is priced higher, "A"s preference grows to $66 \%$, and reduces to $45.5 \%$ when a third option is introduced, although "A" remains the cheapest (Scenarios 5 and 6).

In Scenarios 7 to 9, the first bundle was changed to include only one change and the second bundle was designed inclusive of both a generous baggage allowance as well as unlimited changes, becoming clearly more appealing than the first bundle. Although now "A" is less attractive than before, it was preferred $67 \%$ of the time due to the price gap of $\$ 50$, and dropped to $45 \%$ of the time in the 3-bundles case. In scenario 9, even when a very generous baggage allowance bundle is introduced, priced 50\% higher than the first bundle, "A"'s preference shrinks by 18 percentage points, which are transferred towards the second and the third option (Fig. 3).

As observed in all cases, adding a third option can nudge respondents towards the second bundle. As the trip purpose was not the topic of the survey, any product-price elasticity related conclusions are avoided by the author. It is worth mentioning however that the economic context has a direct influence on such results: a pandemic increases the perceived utility of flexibility, even though purchases are done much closer to the travel dates.

\section{Branding and choices}

In order to streamline options and to easily differentiate offers, airlines have designed specific sets of product characteristics, called branded fares. Branded fares are marketed by airlines as product bundles, combining fares with a set of ancillary services or fare restrictions ("Economy Basic" or "Economy Flex" as an example) which can help the consumer to understand what products the airline is able to offer.

The brand names themselves can influence myopic behavior. Picking the right brand name for a new product is of utmost importance as the first thing a customer learns about a new product is its name. It is expected that initial designs of the choice architecture such as the name and number of brands, the product characteristics that are grouped together in each brand and the price ranges used in each brand will all be suboptimal when first implemented by an airline. This means that the airline must be ready to learn and unlearn from consumer behaviours and conduct experiments and observe the effectiveness of its fare brands. Price experimentation methods such as Thompson sampling in the multiarmed bandit method can be particularly useful in pricing the offers.

Although the next generation revenue management systems have to perform a total offer management, including pricing the offers at the level it deems appropriate, the consumers must perceive a simple display and an easy interaction with the offer management system. Airlines will have to invest in dynamic offer generation models that will output very few options in real time-the ones that are the most likely to appeal to the consumer originating the request. A set of offers which are too complex can lead to choice overload, where consumers become reluctant to make a purchase because of the overwhelming number of options (Park and Jang 2013). Choice overload can even influence people to switch to a brand that is simpler, with less options. In general, people enjoy having choices, but they experience stress if they are exposed to too many, leading to poor choices or no purchase. The potential for regret also goes up with too many choices that are complicated to analyse, consumers wishing they had chosen something slightly different. Many choices can lead people to compare attributes they 


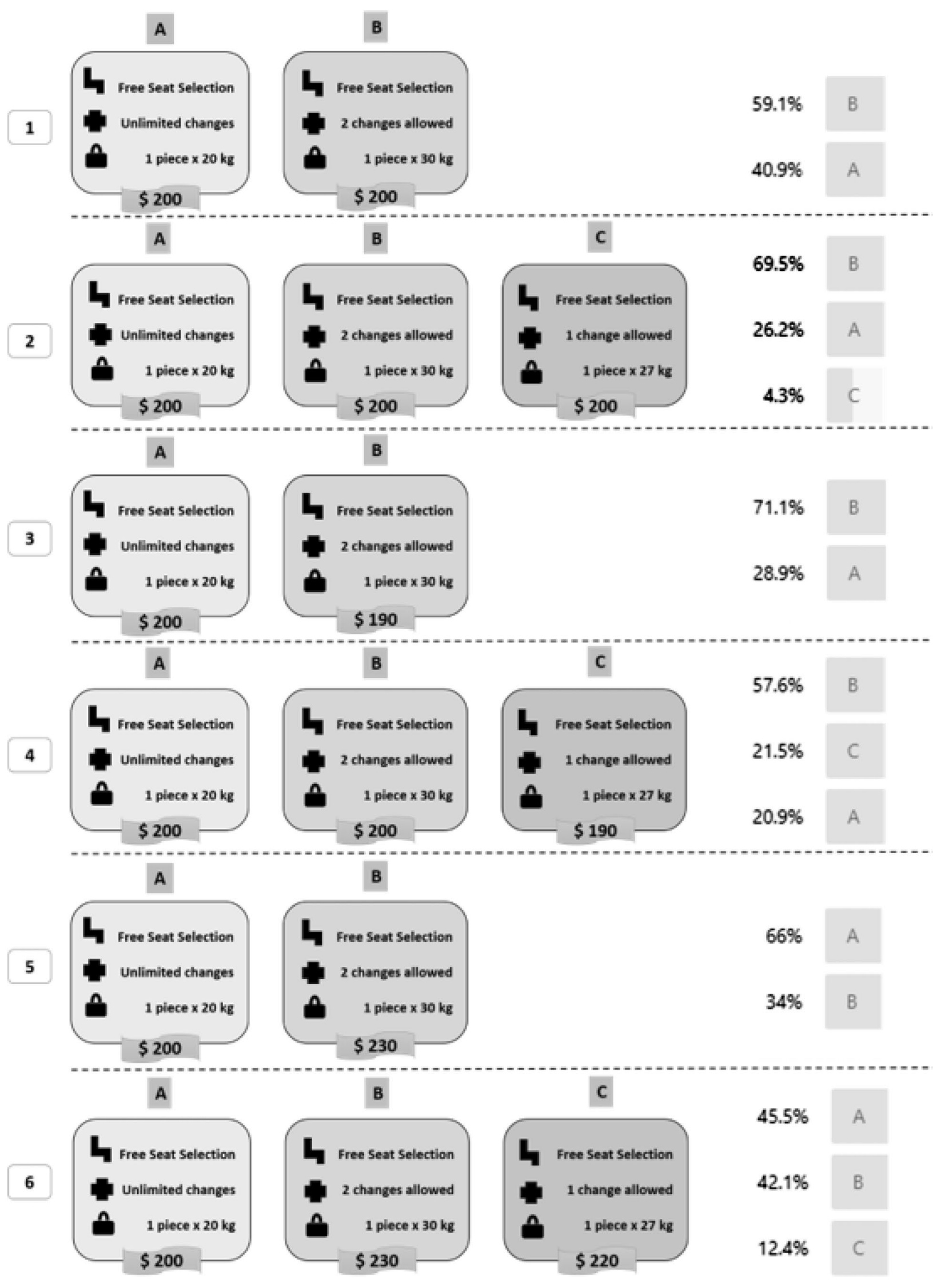

Fig. 2 Survey results 1-6 


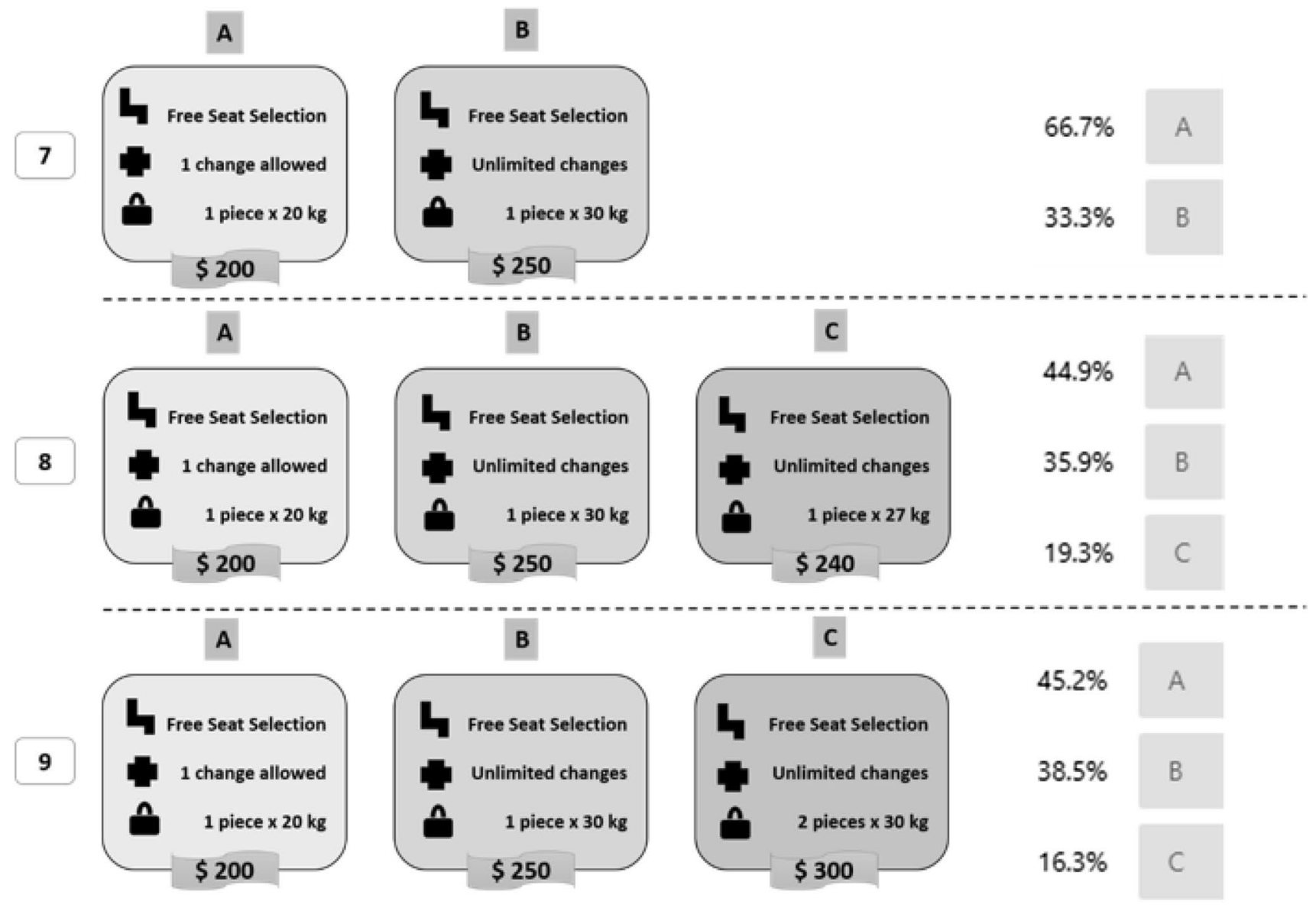

Fig. 3 Survey results 7-9

don't necessarily value, and they lose sight of what really matters to them in the process, making them unhappy. Most retailers tend to display a maximum of three options and in many cases the middle option is the one preferred-such as a medium sized cup at a coffee shop. In the case of choice overload however, people choose the bare minimum, the full option, the "most popular"/what is recommended or they simply don't pick any option.

Even when the choice set is simple, the display environment and the temporal context plays a crucial role in ensuring customer engagement. When thinking about choices for the long term future, people tend to make wise choices, but when asked to make the same choices today, a more impulsive behavior is observed, driven by instant gratification. This can be exploited and the cues (environment, context) can influence/nudge human behaviour into, or out of, instant gratification (Cheng et al. 2012).

Despite all these elements, organisations tend to spend too much time on the first mile- the initial phases of a project-and way too little on the last mile-delivery/ deployment of the product/service to the market. Instead of adding significant time and resources to analyse the way their product is perceived by consumers, many companies already prepare the next best thing, the next product/ service (Soman 2015). Constantly changing the products and services can also be detrimental for airlines. Once fare brands and ancillaries are defined it is advisable that airlines adjust their prices or some of their characteristics, rather than drastically changing the brands consumers are used to seeing. In this complex world, the secret to obtaining incremental revenue by fine tuning offers and addressing consumer needs remains experimenting and allowing models to explore, rather than only to exploit the available data sets (Oancea 2020).

In summary, although sophisticated techniques can be used by the offer engines, airlines need to be clever enough to keep things simple for the consumer. A $20 \%$ discount is more persuasive than a $20.37 \%$ discount and despite the multitude of customer preferences, choices should be streamlined to keep only those that people value. Offers must be well organised and displayed, as consumers will ignore them if they appear to have too much information or they seem too complex to analyse. 


\section{Future implications and conclusion}

The new world of offers comes with a lot of challenges for airlines and system providers. Data privacy compliance of the new models built will have to be addressed, but equally, when a consumer is willing to share tremendous amounts of data in the hope of an easy and personalised process, airlines nowadays fail to adequately respond to the needs expressed directly or implicitly by the consumer. The pandemic impact has seen each airline react in its own way, some being more agile than others in adjusting capacities and pricing structures in response to recent consumer behaviours. This highlighted the need of accelerating the science evolution of the pricing function, enlarging its scope towards enriched offer creation. True dynamic pricing of bundles and adaptive models of customer segmentation can have a key contribution to revenue success, particularly in rapid demand fluctuation scenarios. Airlines will have to constantly evolve their offerings and re-price the features based on the choice behaviours they observe. This means fundamental changes not just to the models used but also to the way airlines are structured in their work processes and the way decision makers-also people-in airlines, interact with these new models to formulate commercial strategies and deliver a seamless and coherent set of services in all customer touchpoints. A culture of experimentation will be key in order to constantly learn from customers and adjust the offer sets and their prices. Artificial intelligence solutions must be trusted and allowed to automatically use a bounded balance of exploration and exploitation to achieve long term revenue improvement, in a world where current bookings classes might become obsolete, the bundling of products might be dynamic and pricing might become entirely continuous.

\section{References}

Bockelie, A., and P. Belobaba. 2017. Incorporating ancillary services in airline passenger choice models. Journal of Revenue and Pricing Management 16 (6): 553-568.
Cheng, Y.Y., P.P. Shein, and W.B. Chiou. 2012. Escaping the impulse to immediate gratification: The prospect concept promotes a future-oriented mindset, prompting an inclination towards delayed gratification. British Journal of Psychology 103 (1): 129-141.

Lu, Y. 2019. Impacts of ancillary services on airline revenue management. Master's Thesis, Massachusetts Institute of Technology.

Nammir, D.S.S., B.M. Marane, and A.M. Ali. 2012. Determine the role of customer engagement on relationship quality and relationship performance. European Journal of Business and Management 4 (11): 27-36.

Oancea, Octavian. 2020. Optimizing airline fare structures. Journal of Revenue and Pricing Management 19 (4, No 3): 230-233.

Park, J.Y., and S.S. Jang. 2013. Confused by too many choices? Choice overload in tourism. Tourism Management 35: 1-12.

Rodway, P., A. Schepman, and J. Lambert. 2012. Preferring the one in the middle: Further evidence for the centre-stage effect. Applied Cognitive Psychology 26 (2): 215-222.

Soman, D. 2015. The last mile: Creating social and economic value from behavioral insights. Toronto: University of Toronto Press.

Thaler, R.H., and C.R. Sunstein. 2009. Nudge: Improving decisions about health, wealth, and happiness. New York: Penguin.

Wang, K.K. 2020. Airline revenue management with dynamic offers: Bundling flights and ancillary services. Doctoral Dissertation, Massachusetts Institute of Technology.

Weaver, R., and S. Frederick. 2012. A reference price theory of the endowment effect. Journal of Marketing Research 49 (5): 696-707.

Welch, John R. 2019. When Econs are human. Journal of Economic Methodology 27 (3): 212-225.

Westermann, D. 2013. The potential impact of IATA's New Distribution Capability (NDC) on revenue management and pricing. Journal of Revenue and Pricing Management 12 (6): 565-568.

Publisher's Note Springer Nature remains neutral with regard to jurisdictional claims in published maps and institutional affiliations. 